\section{DIAGNÓSTICO SITUACIONAL DE LAS INMUNODEFICIENCIAS PRIMARIAS: APROXIMACIÓN PRELIMINAR}

\author{
SITUATIONAL DIAGNOSIS OF PRIMARY \\ IMMUNODEFICIENCIES: PRELIMINARY \\ APPROXIMATION
}

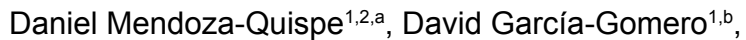 Wilmer Córdova-Calderón ${ }^{3,4, b}$}

Sr. Editor. Las inmunodeficiencias primarias (IDP) engloban un conjunto de enfermedades de la función del sistema inmunitario que predisponen a un incremento de la frecuencia y severidad de infeccioAnes, y a una desregulación del mismo con manifestaciones autoinmunitarias y malignidad ${ }^{(1)}$. La prevalencia de las IDP se estima en 1 en 2000 nacimientos vivos, y se han identificado más de 300 trastornos distintos, entre inmunodeficiencias humorales, inmunodeficiencias combinadas severas y trastornos resultantes de defectos fagocíticos y de complemento ${ }^{(2)}$.

El diagnóstico de IDP aún representa un reto en muchos países de Latinoamérica, incluido el Perú, donde tan solo se reporta 1 caso por cada 516000 habitantes. Sin embargo, se esperaría aproximadamente 300 casos de IDP por año, según la prevalencia global y la expectativa de nacidos vivos en el Perú ${ }^{(3)}$. En Latinoamérica, los principales desafíos relacionados a este subdiagnóstico son a) bajo nivel de conocimiento de IDP entre los profesionales de salud, b) escasos centros de referencia, y c) carencia de laboratorios capaces de realizar pruebas moleculares y genéticas en inmunología ${ }^{(3)}$.

En tal sentido, nos planteamos hacer una aproximación preliminar al diagnóstico situacional de las IDP en nuestro país. Para ello, realizamos una encuesta semiestructurada y autoadmnistrada a 281 profesionales de salud (internos de medicina 40,9\%, médicos

\footnotetext{
Facultad de Medicina. Universidad Nacional Mayor de San Marcos (UNMSM). Lima, Perú.

2 Asociación para el Desarrollo de la Investigación en Ciencias de la Salud (ADIECS). Lima, Perú.

3 Centro de Referencia Nacional en Asma, Alergias e Inmunología. Instituto Nacional de Salud del Niño. Lima, Perú

4 Sociedad Peruana de Inmunología (SPI). Lima, Perú.

a Médico cirujano; ${ }^{\mathrm{b}}$ médico inmunólogo clínico alergólogo.

Recibido: 10/02/2017 Aprobado: 08/03/2017 En línea: 31/05/2017
}

Citar como: Mendoza-Quispe D, García-Gomero D, CórdovaCalderón W. Diagnóstico situacional de las inmunodeficiencias primarias: aproximación preliminar. Rev Peru Med Exp Salud Publica. 2017;34(2):346-7. doi: 10.17843/rpmesp.2017.342.2687 residentes $37,5 \%$ y médicos asistentes 19,2\%) que asistieron a una charla sobre IDP, en 13 hospitales de tercer nivel de Perú ( 8 en Lima, 2 en Arequipa, 2 en Trujillo y 1 en Lambayeque). La mediana de edad fue 28 años. Los resultados se muestran en la Tabla 1. Para el reporte de datos, se incluyeron 281 de 300 encuestas (tasa de rechazo $=6,3 \%$ ) y se excluyeron los valores perdidos (ver el total para cada pregunta en Tabla 1).

El $42 \%$ reportó haber identificado algún caso de IDP; sin embargo, solo el $10 \%$ conoce el registro LASID (Latin American Society for Immunodeficiencies) y tan solo tres de ellos han reportado alguna vez un caso a dicho sistema. LASID es un registro creado para conocer el estado de las IDP en los países de Latinoamérica, entre los cuales se incluye a Perú ${ }^{(3)}$. En este registro, Perú solo contribuyó con aproximadamente $1,6 \%$ de casos reportados a través de cuatro centros de referencia ${ }^{(4)}$. En el Ministerio de Salud, ente rector de las políticas de salud, solo se dispone del Centro de Referencia Nacional de Asma, Alergias e Inmunología, desde hace cuatro años, para atención de pacientes menores de 18 años, quedando ausente la cobertura a pacientes fuera de este rango de edad.

Tabla 1. Respuestas de los profesionales de la salud sobre la situación de las inmunodeficiencias primarias en 13 sedes hospitalarias de Perú

\begin{tabular}{|c|c|}
\hline Preguntas & $n / N^{*}(\%)$ \\
\hline \multicolumn{2}{|l|}{ Sobre el diagnóstico } \\
\hline ¿Identificó algún caso de IDP? & $107 / 255(42,0)$ \\
\hline ¿Conoce el registro LASID? & $28 / 281(10,0)$ \\
\hline ¿Reportó un caso a LASID? & $3 / 281(1,1)$ \\
\hline \multicolumn{2}{|l|}{ Sobre los exámenes auxiliares } \\
\hline ¿Su hospital cuenta con dosaje de lg? & $148 / 262(56,5)$ \\
\hline $\begin{array}{l}\text { ¿Su hospital cuenta con citometría de } \\
\text { flujo? }\end{array}$ & $49 / 249(19,7)$ \\
\hline \multicolumn{2}{|l|}{ Sobre el tratamiento } \\
\hline ¿Indicó o gestionó algún TPH? & $17 / 265(6,4)$ \\
\hline ¿Cuenta con Ig humana disponible? & $83 / 228(36,4)$ \\
\hline ¿Indicó o sugirió alguna vez Ig humana? ${ }^{\dagger}$ & $105 / 281(37,4)$ \\
\hline Sí, en enfermedad de Kawasaki & $41 / 105(39,0)$ \\
\hline Sí, en síndrome Guillain-Barré & $35 / 105(33,3)$ \\
\hline $\begin{array}{l}\text { Sí, en púrpura trombocitopénica } \\
\text { inmune }\end{array}$ & $22 / 105(21,0)$ \\
\hline Sí, en IDP & $17 / 105(16,2)$ \\
\hline
\end{tabular}

IDP: inmunodeficiencia primaria, LASID: Latin American Society for Immunodeficiencies, Ig: Inmunogloblulina, TPH: trasplante de precursores hematopoyéticos

* Se encuestaron 281 profesionales de la salud. Sin embargo, para el reporte de datos se excluyeron los valores perdidos, por lo que el total puede variar en cada pregunta.

† Podía responder más de una opción 
El diagnóstico de IDP se apoya en exámenes como dosaje de inmunoglobulinas y citometría de flujo para el recuento de linfocitos T (CD3, CD4, CD8), B (CD19), NK (CD16/56), entre otros ${ }^{(1,2)}$; sin embargo, vemos que solo el $56,5 \%$ cuenta con dosaje de inmunogloblulinas y el $19,7 \%$ cuenta con citometría de flujo, en sus sedes hospitalarias. Aun cuando contamos con pocos centros que realizan exámenes para el diagnóstico inmunológico, no contamos con pruebas más precisas, como las subclases de inmunoglobulinas, anticuerpos específicos para antígenos bacterianos (ej. neumococo), pruebas de secuenciamiento genético, entre otras.

Para el paciente diagnosticado de IDP existen tratamientos disponibles que han mejorado su esperanza y calidad de vida ${ }^{(1)}$. Entre ellos, remarcamos el uso de inmunoglobulina humana, utilizada en deficiencias primarias de anticuerpos, inmunodeficiencias combinadas antes del trasplante $y$ hasta que se restablezca la función de las células $B$ y en otros trastornos específicos ${ }^{(2)}$, y el trasplante de precursores hematopoyéticos (TPH) indicado para inmunodeficiencias severas que son letales en los primeros años de vida ${ }^{(5)}$. EI $37,4 \%$ de encuestados refirió haber usado inmunoglobulina en alguno de sus pacientes, y tan solo el $6,4 \%$ usó TPH; estos datos reflejan una brecha en el acceso a estos tratamientos. Además, la enfermedad en la que más se usó inmunoglobulina fue la enfermedad de Kawasaki, y solo en cuarto lugar se uso en las IDP.

Nuestra comunicación aproxima un escenario que permite sustentar políticas que busquen mejorar el subregistro de casos, la capacitación del personal de salud para reconocer las IDP, ampliar la contribución de nuestro país al registro LASID, motivar la implementación de laboratorios para diagnóstico inmunológico, y con todo ello lograr la mejora en la calidad y esperanza de vida de las personas que viven con IDP.

Fuentes de financiamiento: autofinanciado

Conflictos de interés: ninguno

\section{REFERENCIAS BIBLIOGRÁFICAS}

1. Bonilla FA, Khan DA, Ballas ZK, Chinen J, Frank MM, Hsu JT, et al. Practice parameter for the diagnosis and management of primary immunodeficiency. J Allergy Clin Immunol. 2015;136(5):1186-205.e1-78. doi: 10.1016/j. jaci.2015.04.049.

2. Picard C, Al-Herz W, Bousfiha A, Casanova J, Chatila T, Conley $\mathrm{M}$, et al. Primary Immunodeficiency Diseases: an Update on the Classification from the International Union of Immunological Societies Expert Committee for Primary Immunodeficiency 2015. J Clin Immunol. 2015;35(8):696726. doi: $10.1007 / \mathrm{s} 10875-015-0201-1$.

3. Costa-Carvalho B, González-Serrano M, EspinosaPadilla S, Segundo G. Latin American challenges with the diagnosis and treatment of primary immunodeficiency diseases. Expert Rev Clin Immunol. 2016;21:1-7. doi: 10.1080/1744666X.2017.1255143

4. Sociedad Latinoamericana de Inmunodeficiencias [Internet]. Estadísticas de LASID enero del 2017 [Citado el 9 de febrero del 2017] Disponible en: https://lasid.org

5. Pai SY, Logan BR, Griffith LM, Buckley RH, Parrott RE, Dvorak CC, et al. Transplantation outcomes for severe combined immunodeficiency, 2000-2009. N Engl J Med. 2014; 371(5):434-46. doi: 10.1056/NEJMoa1401177.

Correspondencia: Daniel Enrique Mendoza Quispe

Dirección: Parque Unión Panamericana 136. Lima, Perú

Teléfono: (551) 987829720

Correo electrónico:daniel91emq@gmail.com

\section{ESTIGMA RELACIONADO A VIH/SIDA Y NO ADHERENCIA AL TRATAMIENTO ANTIRRETROVIRAL}

\section{STIGMA RELATED TO HIVIAIDS AND ADHERENCE TO ANTIRETROVIRAL THERAPY}

\section{Claudia Barreda-Velit ${ }^{1, a}$, Rodrigo Salcedo-Pereda ${ }^{1, a}$}

Sr. Editor. Hemos leído atentamente el estudio realizado en el Hospital Nacional Dos de Mayo en el año $2014^{\left({ }^{(1)} \text {. Nos }\right.}$ resulta relevante la asociación encontrada entre el estigma relacionado a VIH/SIDA y la no adherencia al tratamiento antirretroviral (TARV); más cuando el Perú alcanzó una falta de adherencia del $35,9 \%$ en 2009 (2). No obstante, creemos que el estudio tiene algunas limitaciones que afectarían la medición de la adherencia al TARV y, por lo tanto, a la fuerza de asociación.

En primer lugar, el desorden por uso de alcohol es una variable importante que influye en el análisis de adherencia al TARV. Se han encontrado prevalencias entre 17 y $29 \%$ de abuso de alcohol en pacientes $\mathrm{VIH}$ positivos (3), y existe asociación entre este desorden y la falta de adherencia al TARV ${ }^{(4)}$. Por este motivo, cuestionamos la ausencia de esta variable en el presente análisis. Dentro de limitaciones se mencionan factores

\footnotetext{
Escuela de Medicina, Facultad de Ciencias de la Salud, Universidad Peruana de Ciencias Aplicadas, Lima, Perú.

a Estudiante universitario de Medicina

Recibido: 17/02/2017 Aprobado: 08/03/2017 En línea: 28/06/2017
}

Citar como: Barreda-Velit C, Salcedo-Pereda R Estigma relacionado a VIH/ SIDA y no adherencia al tratamiento antirretroviral. Rev Peru Med Exp Salud Publica. 2017;34(2):347-8. doi: 10.17843/rpmesp.2017.342.2699 